\title{
Microfluidic Devices for Behavioral Screening of Multiple Zebrafish Larvae: Design Investigation Process
}

\author{
Arezoo Khalili ${ }^{1}$, Ellen van Wijngaarden ${ }^{1}$, khaled Youssef $^{1}$, Georg Zoidl ${ }^{1}$, and Pouya Rezai ${ }^{1}$ \\ ${ }^{1}$ York University
}

February 11, 2021

\begin{abstract}
Microfluidic devices have been introduced for phenotypic screening of zebrafish larvae in both fundamental and pre-clinical research. One of the remaining challenges for the broad use of microfluidic devices is their limited throughput, especially in behavioural assays. Previously, we introduced the tail locomotion of a semi-mobile zebrafish larva evoked on-demand with electric signal in a microfluidic device. Here, we report the lessons learned for increasing the number of specimens from one to four larvae in this device. Multiple parameters including loading and testing time per fish and loading and orientation efficiencies were refined to optimize the performance of modified designs. Simulations of the flow and electric field within the final device provided insight into the flow behavior and functionality of traps when compared to previous single-larva devices. Outcomes led to a new design which decreased the testing time per larva by approximately $60 \%$. Further, loading and orientation efficiencies increased by more than $80 \%$. Critical behavioural parameters such as response duration and tail beat frequency were similar in both single and quadruple-fish devices. The optimized microfluidic device has significant advantages for greater throughput and efficiency when behavioral phenotyping is required in various applications, including chemical testing in toxicology and gene screening.
\end{abstract}

\section{Hosted file}

Device Design Paper, AK14 EW14 KY1 PR7 GZ2.pdf available at https://authorea.com/users/ 395206/articles/508526-microfluidic-devices-for-behavioral-screening-of-multiple-

zebrafish-larvae-design-investigation-process 